\title{
An architectural framework for automatic detection of autism using deep convolution networks and genetic algorithm
}

\author{
Nagashree Nagesh ${ }^{1}$, Premjyoti Patil ${ }^{1}$, Shantakumar Patil ${ }^{2}$, Mallikarjun Kokatanur ${ }^{3}$ \\ ${ }^{1}$ Department of Computer Science and Engineering, Nagarjuna College of Engineering and Technology, Bengaluru, India \\ ${ }^{2}$ Department of Computer Science and Engineering, Sai Vidya Institute of Technology, Bengaluru, India \\ ${ }^{3}$ Xebia IT Architects Pvt Ltd., Bengaluru, India
}

\begin{tabular}{l} 
Article Info \\
\hline Article history: \\
Received Mar 4, 2021 \\
Revised Sep 16, 2021 \\
Accepted Oct 11, 2021 \\
\hline
\end{tabular}

Keywords:

Affine transformation Autism spectrum disorder Convolution neural networks Genetic algorithm Magnetic resonance imaging

\begin{abstract}
The brainchild in any medical image processing lied in how accurately the diseases are diagnosed. Especially in the case of neural disorders such as autism spectrum disorder (ASD), accurate detection was still a challenge. Several noninvasive neuroimaging techniques provided experts information about the functionality and anatomical structure of the brain. As autism is a neural disorder, magnetic resonance imaging (MRI) of the brain gave a complex structure and functionality. Many machine learning techniques were proposed to improve the classification and detection accuracy of autism in MRI images. Our work focused mainly on developing the architecture of convolution neural networks (CNN) combining the genetic algorithm. Such artificial intelligence (AI) techniques were very much needed for training as they gave better accuracy compared to traditional statistical methods.
\end{abstract}

This is an open access article under the CC BY-SA license.

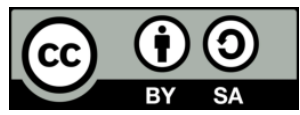

\section{Corresponding Author:}

Nagashree Nagesh

Department of Computer Science and Engineering, Nagarjuna College of Engineering and Technology

Bangalore, Karnataka, India

Email: astrodamsel@gmail.com

\section{INTRODUCTION}

Autism spectrum disorder (ASD) is a disability in nervous group of human brain which causes a lack of social communication, repetitive behavior, and heterogeneity in its nature which ranges from person to person. Due to its high ubiquity, many researchers have turned towards the latest Artificial Intelligence and machine learning (ML) techniques to analyze rather traditional statistical methods [1]. ASD generally affects the human brain and results in trouble in speech, interaction with society, and communication problems and also delays in kinetics. This can be diagnosed with experts from age of 2 years onwards or still before that in very recent times [2]. Many deep learning techniques show pretty good performance in challenging artificial intelligence (AI) and ML tasks. Generally, most of the convolution neural networks (CNN) architecture contains several convolutions, pooling, and fully connected layers. Several architectures have been discovered to achieve classification accuracy. Many architectures have been proposed in the literature, for example, GoogleNet [3], and RESONET [4]. Though many architectures are quite efficient in identification and classification processes, still researchers are finding better ways to improvise classification accuracy [5]. Nowadays, deep learning technology has been widely used for analyzing medical images as in recent years it has gained a lot of popularity and given excellent performance in various fields. Among all the deep learning approaches, CNN is the best solution always for large-scale image classification applications.

In this paper, we discuss an architectural framework for CNN including an evolutionary optimization approach i.e., Genetic algorithm for training the model. Multiple convolution layers are considered and each step of the genetic algorithm is represented to be the CNN nodes [6]. Individual 
generation in standard evolutionary algorithms contains genetic operators such as selection, crossover, and mutation. The CNN architecture is developed from scratch to increase more competitiveness. According to the survey, one in seventy children is pretentious by autism. In 2018, the ubiquity of ASD was around 168 out of 10,000 in the United State. Due to its high prevalence nature, many are turning towards Artificial Intelligent approaches to give the solution for it. Many articles were included in this review, with several different supervised machine learning methods like support vector machine (SVM), LASSO, random forest, linear discriminant analysis. Deep learning methods are used in deep neural networks as a prototype shown better performance different AI and ML tasks such as recognition of images [5]-[7], speech recognition [5], and reinforcement learning tasks [8]. CNNs have given great success in image recognition and are put into several computer vision implementations. Evolutionary optimization algorithms have been traditionally applied in designing neural network architectures [9]. Generally, there are two types of encoding systems: direct and indirect. Direct coding measures the number and connectivity of neurons directly as the genotype and indirect coding is where it contains an occasioning set of regulations for network architectures.

Though classical approaches optimize the number and connectivity of low-level neurons, the latest architectures for $\mathrm{CNN}$ have multiple units like convolution, pooling, and normalization. There is quite a reasonable amount of computation involved in optimizing the networks, therefore training of nodes in the neural network unit is promising. The $\mathrm{CNN}$ research has witnessed a breakthrough since the introduction of the Alex Net network [3]-[9]. In recent years there has been a tremendous improvement in CNN for largescale image classification [10]. Due to advances in CNN components [8]-[10] and training strategies [10], [11], our approach achieves better recognition accuracy using architectural alterations that contain adapting existing CNNs into a new architecture framework [3], [12]. The basic architecture of Image classification using CNN model is illustrated in Figure 1.

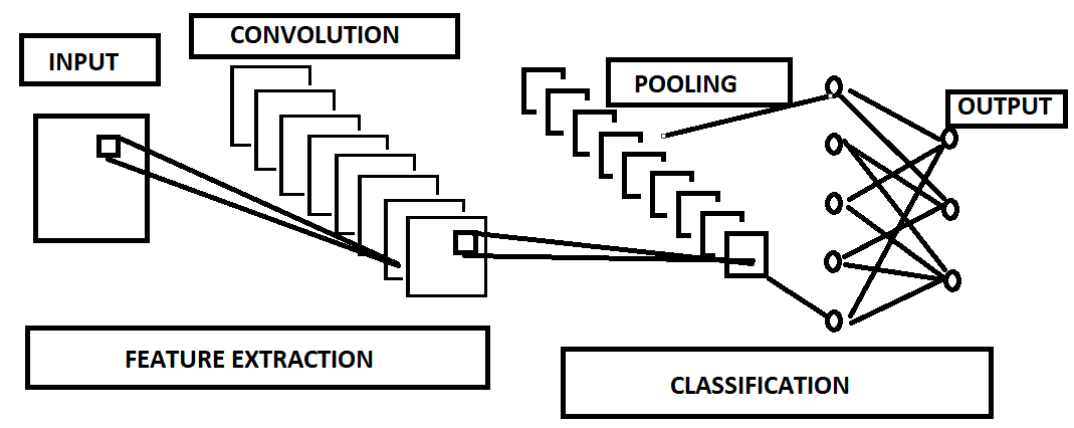

Figure 1. Basic CNN model for Image classification model

In [7]-[9], training of the network is achieved from a large capacity of CNN over the traditional neural network approach for classification problems. This kind of network does not require extensive training of base CNN models over other traditional models. Most of the networks seen in literature have witnessed training by the divide and conquer method. In contrast to the above, the architectures in [5], [6], [10], [11] consider input images to only a selected by experts which increases the recognition accuracy. Bala and Yasmin [6] in their research conclude fine-tuning of the complete network in the final phase of training. It is possible to better improvise training accuracy but in practice, it was not done because of large computational cost.

In [13], research is conducted to experiment with metrics on various segmentation methods of autistic brain. A new matric is developed to identify the goodness of segmentation algorithms. A genetic thresholding-based algorithm is introduced in [14] for MRI image segmentation to detect autism and dementia in magnetic resonance imaging (MRI) image. Recently CNN have been evolved significantly for MRI as well as fMRI brain images towards automatic detection of Autism [15], [16]. Eigen values detection from the feature points extracted from the brain images were also one of the factors for automatic autism detection. The method would employ latent dirichlet allocation (LDA) and other statistical classifier methods. With this feature discriminant analysis around $77 \%$ of accuracy in autism detection is identified [17], whereas, in contrast to statistical methods machine learning algorithms had given much better accuracy which is proved in a thesis [18].

Image segmentation is very crucial step in classification and detection of autism. However, there are many challenges associated in segmenting a brain image. Also due to swift development in medical imaging, different segmentation algorithm has to be proposed for specific imaging applications. There are too many 
challenges involved in image segmentation which are highlighted in [19]. Hyde et al. [20] in his review article has proposed how supervised machine learning is applied in ASD. This paper provides a comprehensive review of around 45 papers including algorithms for classification. Since autism is a behavioral disorder, structural as well as functional brain analysis is essential in planning any classification algorithm. Chen et al. in his recent publication, reviewed recent advances in understanding structure of brain and analyzed why structural analysis plays a significant role [21]-[23]. As discussed in many articles, feature detection is the very essential step towards autism detection. Initial step involves Haar feature detection which was employed for facial feature detection [24], [25]. In most of the literature, many machines learning and deep learning approaches have been proposed which uses ABIDE dataset for acquiring autistic MRI images. An efficient neural network classifier is used [26]-[28].

\section{RESEARCH METHOD}

The main objective of this paper is to develop architecture for CNN by combining steps of genetic algorithm towards automatic detection of autism. The objectives of this work include the following.

\subsection{Population}

To represent a population of chromosomes in a genetic algorithm and each chromosome is a node in CNN. Every MRI brain image is regarded as an individual node in convolution layers of CNN. Hence, they are regarded as population. This step is further subjected to feature extraction to detect important features of each image in a given population. Finally, a 2D matrix of all the feature points extracted are represented as multiple layers in CNN processing. This phase results into feature mapping level, the result of feature mapping as shown in Figure 2.

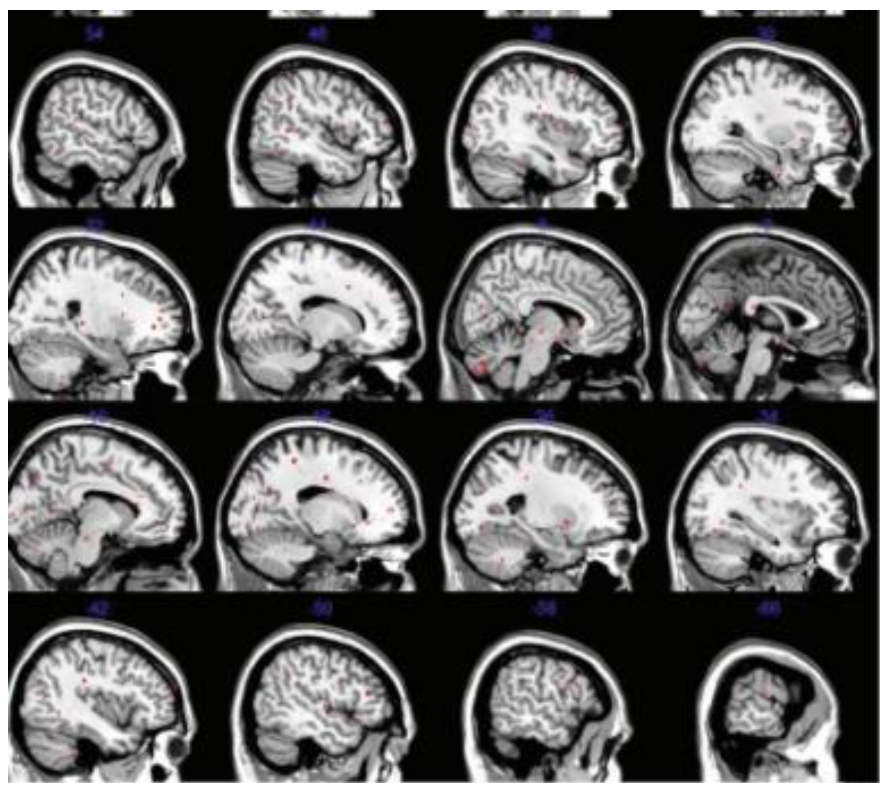

Figure 2. Feature map of brain image [12]

\subsection{Selection}

To develop a selection criterion to choose a best fit point for Genetic algorithms (GA) further processing with respect to CNN layer. The genetic selection works on the principle of Survival is the fittest. Accordingly, a fitness function is derived to perform Image Registration. Each matrix is represented as a corner map. This approach incorporates Image registration process to align the MRI images into its standard orientation which involves finding Curvature Scale Space over a given image. Thus, a selection function is represented as in (1).

$$
\theta=\operatorname{Inv} \operatorname{Cos}\left\{\frac{(x 1-x 2) *(y 1-y 2)}{(x 3-x 2) *(y 3-y 2)}\right\}
$$


The equation (1) is derived to accurately align the images. Since trigonometric cosine of an angle is considered the name inverse cosine (InvCos) [15]. Figure 3 shows selection operation where the image registration is considered as fitness function.

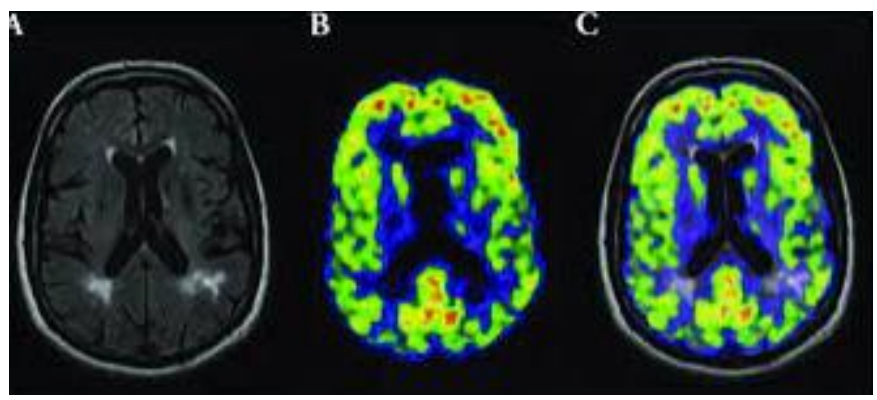

Figure 3. CNN model for registration of images

\subsection{Genetic operators}

To propose an effective genetic operator such as crossover and mutation. Here the segmentation of corpus callosum, white gray matter and cerebrospinal fluid is represented as a part of image segmentation. After segmenting area of corpus callosum (CC), white matter (WM), gray matter (GM) and cerebrospinal fluid (CSF) are calculated. In this step, a colonial hopping algorithm is implemented to find the area of 2D brain covered by corpus callosum, white and gray matter. After segmenting important features of brain such as Corpus Callosum, their individual feature points are extracted to make an area by adding up all points. The area finding function is as shown in (2):

$$
\text { Surface_Area }=2\left(\pi * \text { radius }^{2}\right)+2(\pi * \text { radius } * \text { height })
$$

Similarly, areas of all images which are subjected to training phase are calculated. In the (3), (4), (5), and (6), CC1, CC2, CC3, indicates areas of segmented corpus callosum of image1, image2, image3, respectively. Similarly, for (3), (4), (5), and (6). Using the (3), (4), and (5) the area of surface is calculated and the result of segmentation is shown in Figure 4.

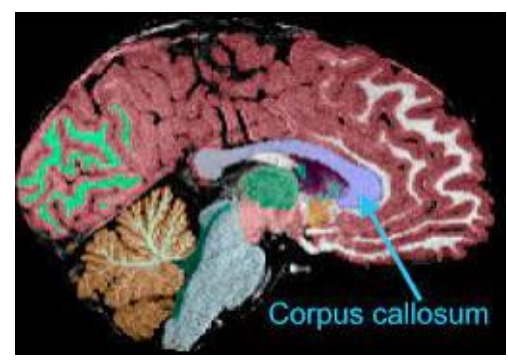

Figure 4. Segmented areas of corpus callosum, white matter and gray matter

$$
\begin{aligned}
& \text { AreaOfCC }=C C^{1}, C C^{2}, C C^{3}, \\
& \text { AreaOfGM }=G M^{1}, G M^{2}, G M^{3} \\
& \text { AreaOfWM }=W M^{1}, W M^{2}, W M^{3} \\
& \text { AreaOfCSF }=C S F^{1}, C S F^{2}, C S F^{3}
\end{aligned}
$$

\subsection{Generation}

To find the evaluator for next iteration (Generation): In the segmentation phase, we have segmented corpus callosum, white and gray matter using colonial hopping algorithm [2] and resulted into areas in the 
training phase. A comparison of all angles was with a threshold value and based on those area, images are classified as autistic and non-autistic images. The general framework to train neural network using convolution network is constructed as in Figure 5.

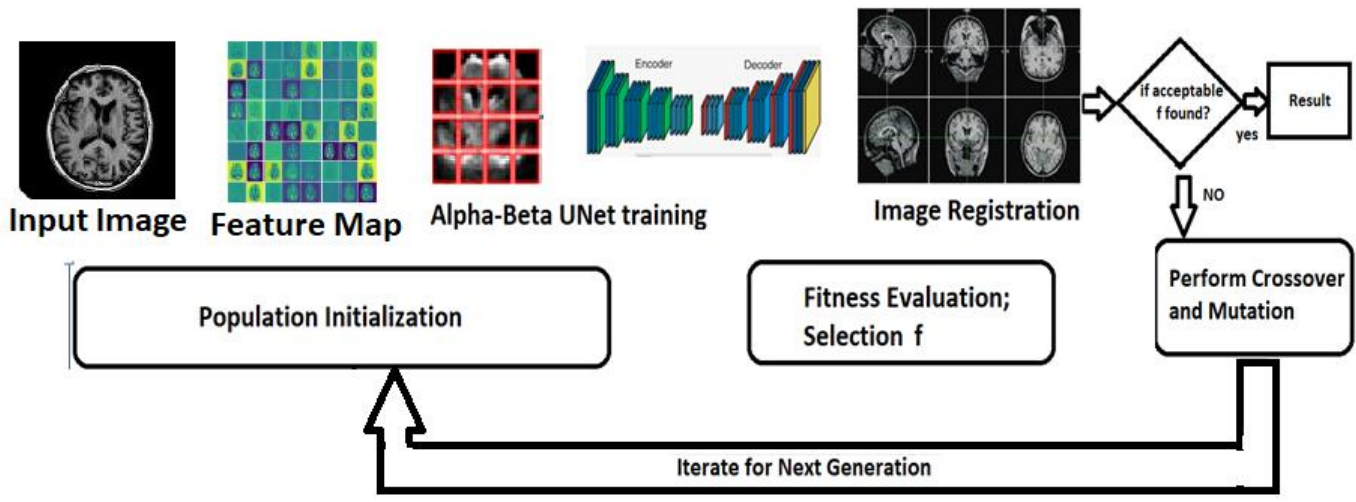

Figure 5. A generalized architecture for our proposed work

\section{RESULTS AND DISCUSSION} with CNN.

The proposed work has following steps involved when applied with genetic algorithm optimization

\subsection{Population initialization}

Around 100 MRI images of brain with 71 autistic and rest normal images are considered as a sample input to train CNN. Each image is independently sampled and corners are extracted. In GA, individual nodes are considered as chromosomes in population at initial stages. Those chromosomes are subjected to feature extraction Figure 6 displays the corner points plotted on MRI brain image using corner extractor operator and corresponding corner graph is shown in Figure 7.

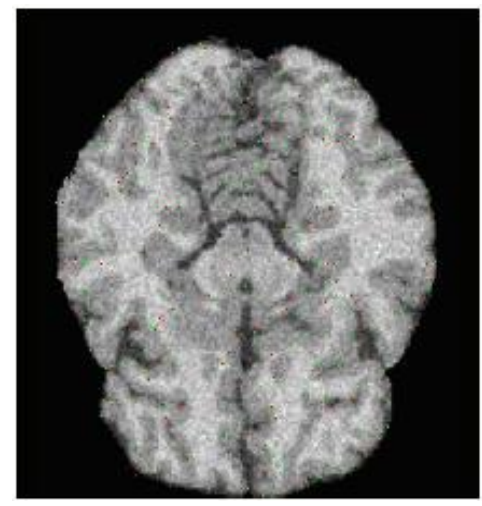

Figure 6. Result of corner map of individual chromosome (MRI image)

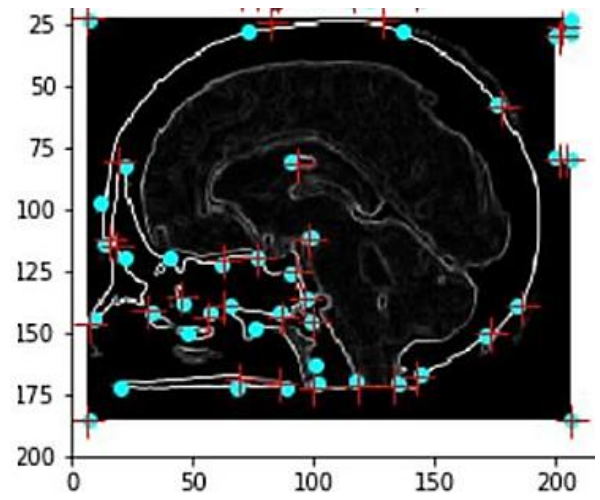

Figure 7. Result of corner extraction map plotted as graph

\subsection{Selection}

Every GA contains selection at the beginning of every generation. Selection is a process to determine which individuals survive. This process works on survival is the fittest policy and we have evaluated the fitness function to select the best fit. Image registration is performed with a fitness evaluation shown in (7) to get the following result. The result of selection operation is illustrated in Figure 8.

$$
\text { dist }=\sqrt{(x 1-x 2) 2+(y 1-y 2) 2}
$$




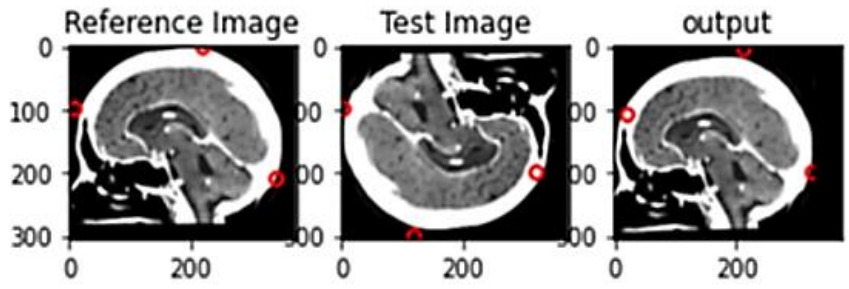

Figure 8. Result of convolution layers for fitness evaluation (selection)

\subsection{Crossover and mutation}

Crossover involves exchanging set of individuals where segmented matrices are extracted on each and every image. In our identified segments a separate area matrix is represented and checked with a threshold to classify how many are autistic images. After applying genetic operators, the area of segmentation is calculated and the Tables 1 and 2 show the areas of segmented regions of autistic and non-autistic brains of corpus callosum, white and gray matter.

Table 1. Segmentation areas of autistic brain

\begin{tabular}{cc}
\hline Part of segmented Brain & Area (In Units) \\
\hline Corpus Callosum & 72 \\
White Matter & 180 \\
Gray Matter & 190 \\
Cerebrospinal Fluid & 250 \\
\hline *generic percentage of autistic images
\end{tabular}

Table 2. Segmentation areas of non-autistic brain

\begin{tabular}{cc}
\hline Part of segmented Brain & Area (In Units) \\
\hline Corpus Callosum & 32 \\
White Matter & 90 \\
Gray Matter & 90 \\
Cerebrospinal Fluid & 50 \\
\hline *generic percentage of non-autistic images
\end{tabular}

\section{CONCLUSION}

Our work focused mainly on architectural framework designing by combining CNN architecture in Genetic algorithms. The main reason to include GA in CNN is to optimize the training phase in neural network when multiple convolutions and pooling layers are considered. As genetic algorithm is known for its evolutionary principle, when combined with deep learning, it has proved to be efficient in training convolution network. Each stage of GA i.e., population, selection, mutation and crossover are carefully selected for fitness evaluator and thus resulted into a finer architecture

\section{REFERENCES}

[1] R. Sil, A. Roy, B. Bhushan, and A. K. Mazumdar, "Artificial intelligence and machine learning based legal application: the stateof-the-art and future research trends," in Proceedings - 2019 International Conference on Computing, Communication, and Intelligent Systems, ICCCIS 2019, Oct. 2019, vol. 2019-Jan, pp. 57-62, doi: 10.1109/ICCCIS48478.2019.8974479.

[2] T. R. P. B.J. Bipin Nair, "A segmentation approaches to detect autism and dementia from brain MRI," International Journal of Recent Technology and Engineering (IJRTE), vol. 7, no. 5C, 2019.

[3] C. Szegedy et al., "Going deeper with convolutions," in Proceedings of the IEEE Computer Society Conference on Computer Vision and Pattern Recognition, Jun. 2015, vol. 07-12-June, pp. 1-9, doi: 10.1109/CVPR.2015.7298594.

[4] E. Abdel-Maksoud, M. Elmogy, and R. Al-Awadi, "Brain tumor segmentation based on a hybrid clustering technique," Egyptian Informatics Journal, vol. 16, no. 1, pp. 71-81, Mar. 2015, doi: 10.1016/j.eij.2015.01.003.

[5] N. N. Nagashree, P. Patil, S. Patil, and M. Kokatanur, "Alpha beta pruned UNet-a modified unet framework to segment MRI brain image to analyse the effects of CNTNAP2 gene towards autism detection," in 2021 3rd International Conference on Computer Communication and the Internet, ICCCI 2021, Jun. 2021, pp. 23-26, doi: 10.1109/ICCCI51764.2021.9486783.

[6] M. Bala and S. Yasmin, "Study the corpus callosum of brain to explore autism employing image segmentation," International Journal of Neuroscience and Behavioral Science, vol. 4, no. 3, pp. 37-44, Dec. 2016, doi: 10.13189/ijnbs.2016.040301.

[7] N. R. S. B.J. Bipin Nair, Gopi Krishna Ashok, "Classification of autism based on feature extraction from segmented brain MRI," B.J. Bipin Nair, Gopi Krishna Ashok, N.R. Sreekumar, vol. 7, no. 5S3, 2019.

[8] Bram van den Bekerom, "Using machine learning for detection of autism spectrum disorder," in Proc. 20th Student Conf. IT, 2017, pp. 1-7. 
[9] R. Mahajan and S. H. Mostofsky, "Neuroimaging endophenotypes in autism spectrum disorder," CNS Spectrums, vol. 20, no. 4, pp. 412-426, Aug. 2015, doi: 10.1017/S1092852915000371.

[10] Hongyoon Choi, "Functional connectivity patterns of autism spectrum disorder identified by deep feature learning," ArXiv, vol. abs/1707.0, 2017

[11] J. A. Nielsen et al., "Abnormal lateralization of functional connectivity between language and default mode regions in autism," Molecular Autism, vol. 5, no. 1, Feb. 2014, doi: 10.1186/2040-2392-5-8.

[12] S. Bieniecki, W. and Grabowski, "Nearest neighbor classifiers for color image segmentation," in Proceedings of the International Conference Modern Problems of Radio Engineering, Telecommunications and Computer Science, 2004., 2004, pp. $209-212$.

[13] M. K. Nagashree N, Premjyoti Patil, Shantakumar Patil, "Performance metrics for segmentation algorithms in brain mri for early detection of autism," International Journal of Innovative Technology and Exploring Engineering, vol. 9, no. 2S, pp. 561-564, Dec. 2019, doi: 10.35940/ijitee.B1401.1292S19.

[14] P. T. R., "A segmentation approach to detect Autism and Dimentia from brain MRI," International Journal of Recent Technology and Engineering (IJRTE), vol. 7, no. 5C, 2019, doi: 10.13140/RG.2.2.19362.99520.

[15] Z. Sherkatghanad et al., "Automated detection of autism spectrum disorder using a convolutional neural network," Frontiers in Neuroscience, vol. 13, Jan. 2020, doi: 10.3389/fnins.2019.01325.

[16] H. Sharif and R. A. Khan, "A novel machine learning based framework for detection of autism spectrum disorder (ASD)," arXiv, vol. arXiv:1903, 2020

[17] S. Mostafa, L. Tang, and F. X. Wu, "Diagnosis of autism spectrum disorder based on eigenvalues of brain networks," IEEE Access, vol. 7, pp. 128474-128486, 2019, doi: 10.1109/ACCESS.2019.2940198.

[18] G. Katuwal, "Machine learning based autism detection using brain imaging," Theses, Rochester Institute of Technology. Mar. 2017, [Online]. Available: https://scholarworks.rit.edu/theses/9422.

[19] I. Despotović, B. Goossens, and W. Philips, "MRI segmentation of the human brain: Challenges, methods, and applications," Computational and Mathematical Methods in Medicine, vol. 2015, pp. 1-23, 2015, doi: 10.1155/2015/450341.

[20] K. K. Hyde et al., "Applications of supervised machine learning in autism spectrum disorder research: a review," Review Journal of Autism and Developmental Disorders, vol. 6, no. 2, pp. 128-146, Feb. 2019, doi: 10.1007/s40489-019-00158-x.

[21] R. Chen, Y. Jiao, and E. H. Herskovits, "Structural MRI in autism spectrum disorder," Pediatric Research, vol. 69, no. 5 PART 2, pp. 63R-68R, May 2011, doi: 10.1203/PDR.0b013e318212c2b3.

[22] S. Raj and S. Masood, "Analysis and detection of autism spectrum disorder using machine learning techniques," Procedia Computer Science, vol. 167, pp. 994-1004, 2020, doi: 10.1016/j.procs.2020.03.399.

[23] R. Tepest, "The meaning of diagnosis for different designations in talking about autism," Journal of Autism and Developmental Disorders, vol. 51, no. 2, pp. 760-761, Jun. 2021, doi: 10.1007/s10803-020-04584-3.

[24] M. B. Harms, A. Martin, and G. L. Wallace, "Facial emotion recognition in autism spectrum disorders: A review of behavioral and neuroimaging studies," Neuropsychology Review, vol. 20, no. 3, pp. 290-322, Sep. 2010, doi: 10.1007/s11065-010-9138-6.

[25] P. Rani, "Emotion detection of autistic children using support vector machine: a review," International Journal of Innovative Science and Research Technology, vol. 6, 2019.

[26] A. S. Heinsfeld, A. R. Franco, R. C. Craddock, A. Buchweitz, and F. Meneguzzi, "Identification of autism spectrum disorder using deep learning and the ABIDE dataset," NeuroImage: Clinical, vol. 17, pp. 16-23, 2018, doi: 10.1016/j.nicl.2017.08.017.

[27] N. Nagashree, P. Patil, S. Patil, and M. Kokatanur, "InvCos curvature patch image registration technique for accurate segmentation of autistic brain images," in Soft Computing and Signal Processing, Springer Singapore, 2022, pp. 659-666.

[28] S. Liang, A. Q. M. Sabri, F. Alnajjar, and C. K. Loo, "Autism spectrum self-stimulatory behaviors classification using explainable temporal coherency deep features and SVM classifier," IEEE Access, vol. 9, pp. 34264-34275, 2021, doi: 10.1109/ACCESS.2021.3061455.

\section{BIOGRAPHIES OF AUTHORS}

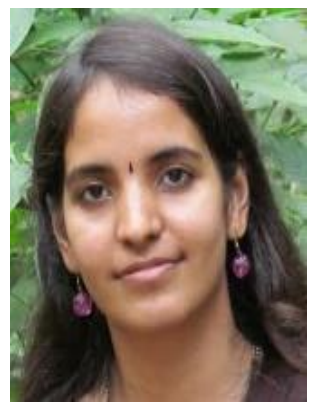

Nagashree Nagesh (iD IS SC P She is working as Asst. Professor in Nagarjuna College of Engineering and Technology. She obtained B. E. (CSE) in 2006 from VTU, and M. Tech (CSE) in 2010 and currently perusing Ph.D, She is having 9+ years of teaching experience, 1 year research experience at Indian Institute of Science, Bangalore. 6 National and International publications, Member of IEEE, Got best paper award in National Conference. She can be contacted at email: astrodamsel@gmail.com.

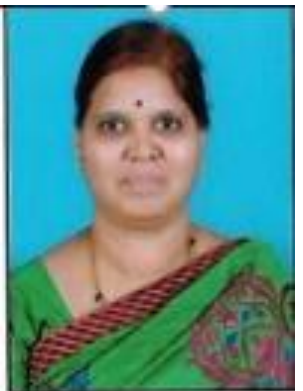

Premajyoti Patil (iD SI SC P She is currently a research supervisor at VTU. She obtained her BE degree in Instrumentation Technology from Gulbarga University, Gulbarga in 1994 and ME degree in Power electronics from Gulbarga University, Gulbarga in 1998 and $\mathrm{PhD}$ in Electrical and Electronics Engineering in Dr. MGR Educational \& Research Institute University, Chennai. She is having 21 years of teaching experience \& she published twenty papers in National and International conferences and guiding two PhD scholars. Her areas of interest are microprocessors, computer networks, computer organization, electronic circuits, and logic design. She has three best technical paper awards in her credit. She is recipient of state level project of the year award during the academic year 2015-2016 conducted by Karnataka State for Science and Technology. She worked as a resource person for summer Internship 2016 on IOT conducted by Texas Instruments University. She is Life member of ISTE and member of IEEE. She can be contacted at email: premjyoti.p@gmail.com.

Int J Elec \& Comp Eng, Vol. 12, No. 2, April 2022: 1768-1775 


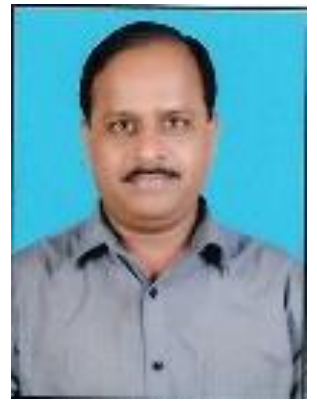

Shantakumar Patil (iD) $8 \mathrm{SC}$ P $\mathrm{He}$ is working as Professor in Sai Vidya Institute of Technology. He obtained his B. E degree in Electrical and Electronics Engineering from Karnataka University Dharwad in 1993 and M. Tech in Computer Science \& Engineering from VTU Belagavi in 2002. He obtained Ph. D degree from Dr. MGR University, Chennai in 2011. His areas of interest are Data Mining, Artificial Intelligence, and Formal Languages \& Automata Theory. He has 24 years of experience in teaching and published twenty Research papers in National/International Journals and conferences. He is recipient of BEST TEACHER award twice, when he was in MVJ College of Engineering and has received BEST PAPER awards in National and International Conferences across the world. He is guiding five Research Scholars for Doctoral Degree. He is Life member of ISTE, CSI and member of IEEE. He can be contacted at email: shantakumar.p@gmail.com.

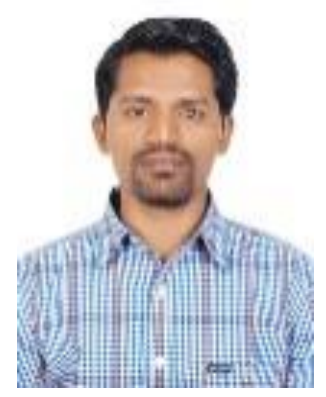

Mallikarjun Kokatanur (D) SC P He is working as Senior Software Engineer in Major IT company and he has obtained B.E(CSE) from VTU in 2006, and MS(IT) from Mangalore University in year 2012. And has 10 years of industrial experience in C, C++, Java, Python, Big Data, NoSQL. He can be contacted at email: mallubk@gmail.com. 